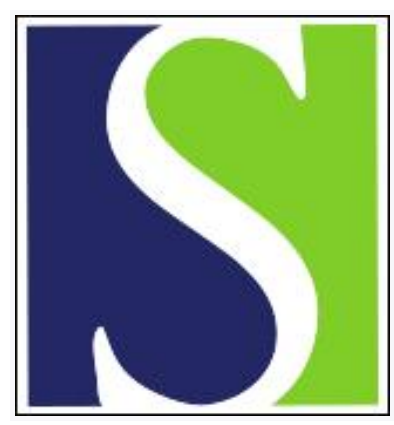

Scand J Work Environ Health 1994;20(1):55-61

https://doi.org/10.5271/sjweh.1428

Issue date: $01 \mathrm{Feb} 1994$

Effect of masking on circadian adjustment and interindividual differences on a rapidly rotating shift schedule.

by Harma M, Waterhouse J, Minors D, Knauth P

Affiliation: Department of Physiology, Finnish Institute of Occupational Health, Helsinki.

This article in PubMed: www.ncbi.nlm.nih.gov/pubmed/8016600

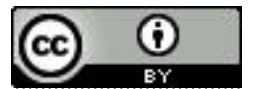




\title{
Effect of masking on circadian adjustment and interindividual differences on a rapidly rotating shift schedule
}

\author{
by Mikko Härmä, MD, ${ }^{1}$ Jim Waterhouse, $\mathrm{PhD},{ }^{2}$ David Minors, $\mathrm{PhD},{ }^{2}$ Peter Knauth, DSc(Tech) ${ }^{3}$
}

\begin{abstract}
HÄRMÄ M, WATERHOUSE J, MINORS D, KNAUTH P. Effect of masking on circadian adjustment and interindividual differences on a rapidly rotating shift schedule. Scand $J$ Work Environ Health 1994;20:55 - 61. OBJECTIVEs - The aim of the present study was to define the effect of masking on the estimation of circadian adjustment of and interindividual differences between nurses on a rapidly rotating shift schedule. MEthods - The phase shift of the circadian rhythm of rectal temperature was studied in 17 subjects. The following three different methods for estimating the circadian phase shift were compared: (i) cosinor analysis, (ii) a method using normative endogenous data in which the masking effects are removed ("purification"), and (iii) a method using normative endogenous data in which the masking effects are not removed. Results - The mean phase delay during the second night shift (compared with the morning shift) was $6.3 \mathrm{~h}$ according to the cosinor analyses of the raw data and $6.2 \mathrm{~h}$ according to the cross-correlation method of the raw data. When the masking effects were removed ("purification"), the phase shift was only $1.7 \mathrm{~h}$. Diurnal type did not significantly explain the differences between individuals in the amount of phase shift of the raw data but was the only significant factor, explaining $32 \%$ of the variation between individuals in the phase shift of the purified data. Conclusions - Masking effects on body temperature should be taken into account before any definite conclusions can be drawn about the relation between individual factors and the adjustment of the circadian rhythm of body temperature.
\end{abstract}

Key terms: body temperature, circadian rhythm, shift work.

The circadian rhythm of body temperature is one of the most commonly used indicators of human circadian rhythmicity. Most earlier field studies on the effects of shift work on this indicator can be criticized on methodological grounds. First, many of them used oral temperature measurements with no or only one data point during the sleep period. (See, eg, references 1-4.) Second, the circadian rhythm of body temperature is dependent on both endogenous and exogenous components. Only the endogenous component reflects the activity of the human biological clock, while the exogenous component depends mainly on environmental and life-style factors like the timing and length of sleep and the amount of physical activity (5). These masking effects on the circadian rhythm of body temperature have not been taken into account in most earlier field studies.

1 Department of Physiology, Finnish Institute of Occupational Health, Helsinki, Finland.

2 Department of Physiological Sciences, University of Manchester, Manchester, United Kingdom.

3 Department of Ergonomics, University of Karlsruhe, Karlsruhe, Germany.

Reprint requests to: Dr M Härmä, Finnish Institute of Occupational Health, Department of Physiology, Laajaniityntie 1, FIN-01620 Vantaa, Finland.
During night shifts the circadian "profile" of the masking effects of sleep and work are dramatically changed compared with that of morning or evening shifts. While the order of the main daily activities during morning shifts is sleep, work, leisure, during night shifts the order is mostly sleep, leisure, work. As has been shown recently in a field study of night work on a slowly retating shift system (6), the circadian adjustment between morning and night shifts is considerably overestimated if masking effects caused by the environment and life-style on body temperature are not removed.

Circadian adjustment to shift work has been reported to be dependent on interindividual differences such as morningness-eveningness (1), introversionextroversion (7), neuroticism (8), and shift-work tolerance $(1,3,4)$, although contradictory results have also been published $(9,10)$. Since the circadian rhythm of body temperature during shift work is greatly influenced by masking, the question arises of whether the interindividual differences reported to explain circadian adjustment are actually explaining the endogenous or only the exogenous influences upon body temperature.

The objectives of the present study were to define the effect of masking on the estimation of circadian adjustment to a rapidly rotating shift schedule and to study the effect of masking on the relation between interindividual differences and circadian adjustment. 


\section{Methods}

\section{Subjects and shift schedule}

After a preliminary questionnaire, 20 women on a rapidly rotating shift schedule and one woman working permanently in night work volunteered for the study. All of the subjects had at least 1.5 years' experience in shift work and were either nurses or nursing aides in a hospital in Kuopio, Finland. From the group working on the rapidly rotating shift schedule, rectal temperature was successfully recorded for 18 subjects. One of these subjects was also excluded since she showed no clear rhythmicity during the second night shift (the percentage rhythm $<10 \%$ ), which precluded useful analysis. The characteristics of the 17 subjects whose data were analyzed are given in table 1 .

The subjects worked a rapidly rotating three-shift system with morning, evening, and night shifts irregularly placed in the shift cycle of three weeks. Morning shifts $(0700-1500)$ and evening shifts $(1300-2100)$ lasted $8 \mathrm{~h}$ and the night shifts $(2100-$ $0700) 10 \mathrm{~h}$. There was an average of $38 \mathrm{~h}$ of work per week, and one shift cycle (three weeks) included an average of seven morning shifts, five evening shifts, and three night shifts. The night shifts occurred mainly as one or two nights in succession. No measurements were accepted during the expected ovulation day and during menstruation, information on which was requested from every subject.

\section{Study design}

Circadian adjustment to night work was estimated from calculations of the phase difference of the body core temperature rhythm from the last morning shift to the second night shift. The following three different methods were used for the phase estimation of the circadian rhythm of body temperature: (i) cosinor analysis of the "raw" (ie, unchanged) data, (ii) analysis of the "raw" data through comparison with normative endogenous data (see the section entitled "Normative Endogenous Data and Purification" and references 5 and 6), and (iii) analysis of the data with the second method but after "purification" to remove

Table 1. Characteristics of the subjects $(\mathrm{N}=17) .\left(\mathrm{VO}_{2} \max =\right.$ maximal oxygen consumption)

\begin{tabular}{lcccc}
\hline Variable & Mean & SD & Range & $\begin{array}{c}\text { Number of } \\
\text { subjects }\end{array}$ \\
\hline Age (years) & 33.9 & 7.2 & $23-48$ & 17 \\
Weight $(\mathrm{kg})$ & 62.9 & 7.5 & $50.0-77.4$ & 17 \\
Height $(\mathrm{cm})$ & 163.1 & 5.6 & $150.5-171.2$ & 17 \\
VO $_{2}$ max $\left(\mathrm{ml}^{\prime} \cdot \mathrm{kg}^{-1} \cdot \mathrm{min}^{-1}\right)$ & 35.0 & 6.8 & $23.8-50.0$ & 17 \\
Diurnal type $^{\mathrm{a}}$ & 2.42 & 0.56 & $1.14-3.29$ & 17 \\
Neuroticism & -0.6 & 3.0 & $-6-+6$ & 17 \\
Extroversion & 1.5 & 2.6 & $-4-+6$ & 17 \\
Living with a partner & & & & 11 out of 17 \\
Children & & & & 10 out of 17 \\
\hline
\end{tabular}

a Morningness, minimum +1 , maximum +6 .

Neuroticism, minimum -6 , maximum +6 .

c Extraversion, minimum -6 , maximum +6 . the masking effects of sleep, work, travel, and strenuous activity.

The effect of masking was analyzed mainly by comparing the shifts assessed by methods 2 and 3. Since many earlier studies have used cosinor analysis of the raw data, this method was also carried out to make comparisons with earlier results possible.

The subjects performed their normal daily routines during the study. The body temperature measurements started at 0700 at the beginning of the first morning shift and ended soon after the second night shift at about 2000 . The morning shift followed another morning shift or a free day, but never an evening or a night shift.

\section{Body temperature and activity recording}

Body temperature was measured every $5 \mathrm{~min}$ by a rectal probe inserted $10 \mathrm{~cm}$ beyond the external anal sphincter. The accuracy of the measurement device was $0.01^{\circ} \mathrm{C}(11)$. (The measuring devices were calibrated by an extreme precise quartz-thermometer of the accuracy of $0.001^{\circ} \mathrm{C}$ ). Yellosprings YSI 400 thermistors were used. No subject was studied on the predicted day of ovulation or during menstruation.

The subjects kept a daily record of the times of the following activities: sleep, leisure, travel, work, and strenuous activity.

\section{Estimation of the circadian rhythm of body temperature}

Cosinor analysis. Cosinor analyses of the raw data were performed according to the single cosinor method (12). The analyses were performed upon hourly data obtained from two 24-h windows starting at about 0800 . Hourly data have been presented instead of data measured every $5 \mathrm{~min}$ so that exactly the same data format can be used for the three different methods of estimating the circadian phase. However, cosinor acrophases were similar whether the 5min or the hourly data were used, with no significant differences between the acrophases.

The first window started at the beginning of the second morning shift, and the second window covered the interval between the end of the first and second night shifts. The phase shift of the rhythm was estimated from calculations of the difference in acrophases between the two windows for each subject.

Normative endogenous data and purification. The method of comparing the "raw" data with normative endogenous data requires hourly readings of rectal temperature, and it has been described in detail elsewhere $(5,6)$. It is based upon the concept of "purification" as used by Wever (13).

The basic concept is that a measured rhythm consists of exogenous and endogenous components that 
act additively. The shape and timing of the exogenous component depend upon several factors, including, mainly, the individual's sleep and activity cycle, sleep lowering the body temperature and different types of activity raising it by different amounts. The endogenous component, whose phase is sought, is assumed to be described by "normative endogenous data." These data are the mean rhythm for the rectal temperature of more than 50 normal subjects (18-25 years of age) studied while awake and in the supine position. Under these conditions, masking effects have been minimized.

The method requires a record of the main type of activity in the previous hour (the choices being, eg, asleep, lying, sitting, standing, walking). The method then "purifies" the observed temperature data according to the following rules: (i) all values during sleep are raised by $\mathrm{A}$, where $\mathrm{A}$ is 0 to $1.0^{\circ} \mathrm{C}$ in $0.1^{\circ} \mathrm{C}$ steps; (ii) all values during lying are unchanged (as this is the posture associated with no masking); (iii) all values during sitting are lowered by $B$, where $B$ is 0 to $1.0^{\circ} \mathrm{C}$ in $0.1^{\circ} \mathrm{C}$ steps; (iv) all values during standing and walking are lowered by $\mathrm{C}$ and $\mathrm{D}$, which are treated as B.

We then tested all combinations of A through D and also, for each combination, shifted the "purified" rhythm by $0-23 \mathrm{~h}$ in hourly steps. The combination of shift and values for parameters A through D which gives the best match with normative endogenous data (ie, minimizes the summed squared deviations between the purified and normative endogenous data) is taken as indicating the shift of the endogenous component in the original data together with the sizes of the masking effects.

Figure 1 gives an example of how the purification worked for one subject. Such an analysis gives (i) a measure of the shift of unchanged (raw) data, (ii) a measure of the shift of changed (purified) data, and (iii) estimates of the masking effects due to sleep, leisure, travel, and work.

\section{Interindividual differences}

The interindividual differences between the subjects were studied with a questionnaire (age, diurnal type, neuroticism, shift-work tolerance, extroversion, marital status, and number of children) and by laboratory measurements of weight, height, and maximal oxygen consumption.

Diurnal type was estimated by the "diurnal type scale" according to Torsvall \& Àkerstedt (14). Diurnal type (morningness-eveningness) correlates with the morning and evening disposition of the sleepwakefulness, as well as with other circadian rhythms of the subjects. Neuroticism and introversion-extroversion were assessed according to Eysenck (15). Shift-work tolerance was estimated with the use of a questionnaire using three criteria: the amount of general fatigue during the last three weeks, the prevalence of digestive disorders, and sleep disturbance (sleep length and quality). A detailed description of the index has been published earlier (16). Marital status was classified as subjects living alone or living with a partner. Maximal oxygen consumption ( $\mathrm{VO}_{2}$ max) was predicted by two different bicycle ergometer tests according to the method recommended by the World Health Organization (17). Real oxygen consumption (Oxycon-4, Mijnhardt, Holland) and the average maximal heart rate for the subjects' age group were used in the prediction.

\section{Results}

The mean circadian phases obtained with the different methods are summarized in table 2. During the day of the morning shift (M), the minimum of the circadian rhythm of the body temperature was near 0400 with no statistical differences in the values obtained with the different methods. During the day of the second night shift (N2), the minimum was delayed more than $6 \mathrm{~h}$ according to the analysis with raw data, but the delay was only $1.65 \mathrm{~h}$ if purified data were used. The phase shift from the morning to the second night shift (M-N2) was highly signifi-

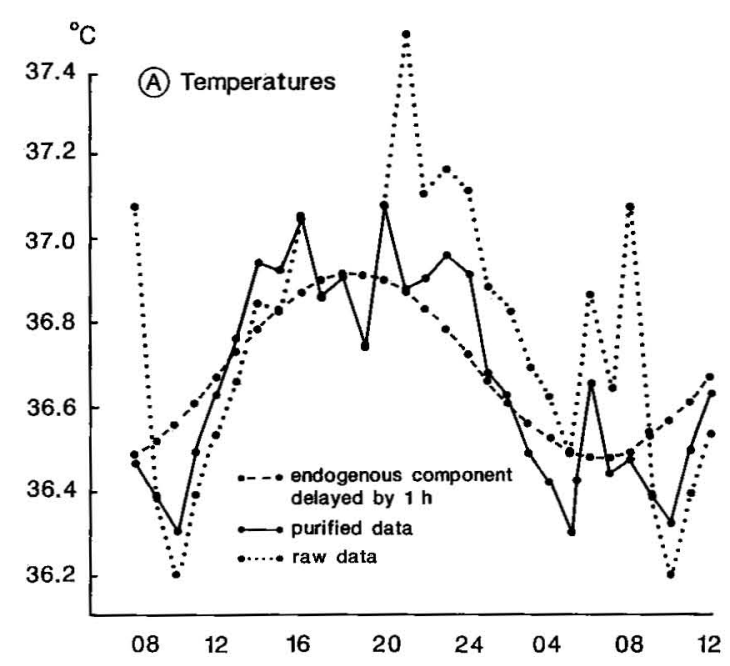

Work

(B) Activity Profile

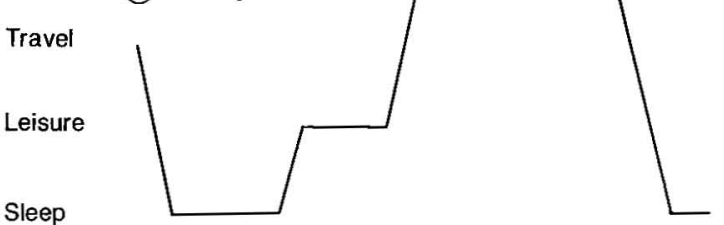

Figure 1. Example of the purification of the temperature data of one subject. [A: temperature curves of the endogenous component delayed by $1 \mathrm{~h}$, purified data and raw data, $\mathrm{B}$ : activity profile (masking effect due to sleep $-0.1^{\circ} \mathrm{C}$, leisure $0^{\circ} \mathrm{C}$, travel $+0.6^{\circ} \mathrm{C}$, and work $+0.2^{\circ} \mathrm{C}$ ] The shift in the raw data is $4 \mathrm{~h}$, and the shift in the purified data is -1 . For other details, see the text. 
Table 2. Circadian phase (decimal time of the minimum) of body temperature during the days including the morning and the second night shifts, the change from the morning to the second night shift, phases from the cosinor analysis and from the method using normative endogenous data using both raw and purified data, and the statistical significance of the phase shift morning-second night shift.

Cosinor method

Normative endogenous method with raw data

Normative endogenous method with purified data

Phase, morning shift (hours)

Phase, second night shift (hours)

Difference in phase or phase shift between the raw and purified data

\begin{tabular}{|c|c|c|c|c|c|c|}
\hline \multicolumn{2}{|c|}{$\begin{array}{l}\text { Phase, morning } \\
\text { shift (hours) }\end{array}$} & \multicolumn{2}{|c|}{$\begin{array}{l}\text { Phase, second } \\
\text { night shift (hours) }\end{array}$} & \multicolumn{2}{|c|}{$\begin{array}{l}\text { Phase shift } \\
\text { (hours) }\end{array}$} & \multirow{2}{*}{ P-value } \\
\hline Mean & SE & Mean & SE & Mean & SE & \\
\hline 4.09 & 0.30 & 10.34 & 0.42 & -6.25 & 0.53 & 0.0001 \\
\hline 3.72 & 0.32 & 9.93 & 0.42 & -6.22 & 0.56 & 0.0001 \\
\hline 3.82 & 0.48 & 5.47 & 0.68 & -1.65 & 0.96 & 0.11 \\
\hline
\end{tabular}

a Purified phase is later.

b Purified phase is not as delayed.

c Purified phase shift is less.

Frequency

10 Distribution of Phase Minimums

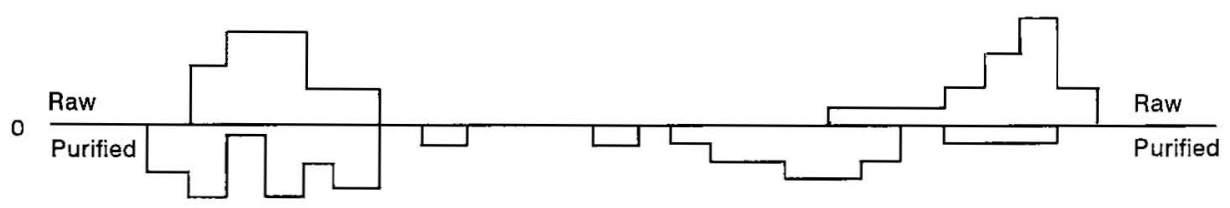

10

Distribution of Midsleep

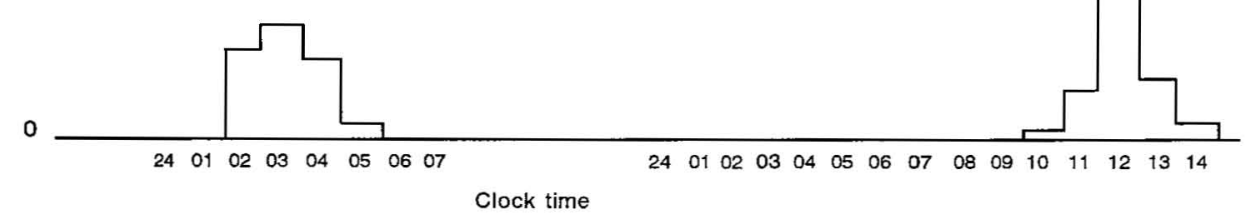

CONTROL

NIGHT 2

Figure 2. Distribution of circadian phase minimums and midsleeps during the days of the morning and second night shifts.

cant when the raw data were used $(\mathrm{P}<0.0001)$ but insignificant $(P=0.1050$, two-tailed $)$ when purified data were used.

These results were almost identical if the cosinor analysis was performed upon the 24 hourly points for each day (the same time points as used with the methods for normative endogenous data) rather than the 2885 -min values. Cosinor analysis also indicated that there was a significant fall of amplitude in the raw data during the second night shift $(\mathrm{P}<0.01)$. Figure 2 shows the distribution of the phase minimums in relation to midsleep (the midpoint of the times of falling asleep and waking up) during the $\mathrm{M}$ and $2 \mathrm{~N}$ shifts. The midsleeps shifted slightly more than the phases of the raw data.

\section{Interindividual differences}

A stepwise regression analysis was performed to study the dependence of the "raw" and "purified" phase shifts on the factors age, weight, height, diurnal type, neuroticism, shift-work tolerance, introversion-extroversion, and physical fitness (maximal oxygen consumption). Since the regression analysis with both analytical methods using raw data produced the same results, only the results of the two methods using normative endogenous data (with raw and purified data) have been reported.

The factors explained $61 \%$ of the variation of the "raw" phase shifts (table 3). Neuroticism explained $18 \%$ of the variation in the phase shifts and had the greatest predictive power, together with height. Neu- 
roticism was not associated with a phase delay in the raw data, the correlation being $-0.43(\mathrm{P}<0.1)$. The correlation between neuroticism and the phase shifts in the cosinor analysis was $-0.42(\mathrm{P}<0.1)$.

The interindividual factors explained somewhat Iess, $46 \%$, of the variation in the phase shifts of the purified data. Diurnal type explained $32 \%$ of the variation and was the most important and also the only significant factor in the regression model. Eveningness was associated with phase delay (figure 3 ), the correlation being significant $(r=0.56, P<0.019)$.

There were some significant correlations between the interindividual factors, and these correlations might have affected the selection of the factors in the regression models. Shift-work tolerance correlated highly with neuroticism $(\mathrm{r}=0.65, \mathrm{P}<0.01)$ and in significantly with age $(r=-0.45, P<0.1)$. The subjects showing poor shift-work tolerance tended to be more neurotic and younger. Shift-work tolerance correlated partly with the phase shift of the raw data (normative endogenous method: $\mathrm{r}=-0.54, \mathrm{P}<0.05$; cosinor method: $r=0.37$, not significant) but did not correlate significantly with the phase shift of the purified data $(r=-0.16$, not significant).

\section{Discussion}

The circadian phase delay of the purified data was $4.6 \mathrm{~h}$ less than the phase delay of the raw data. The change in the cosinor acrophase was also very similar to the phase delay of the raw data assessed by the method using normative endogenous data. Our study confirms the findings of Minors \& Waterhouse (6) that conventional methods which do not remove the masking effects of body temperature greatly overestimate the amount of circadian adjustment during night shifts. The probable explanation is that sleeping and waking were delayed on the night shift, coupled with the fact that most activity took place (during the night work itself) in the second half of the waking span rather than in the first half on the morning shifts. As a further consequence there would also be a lack of phase coincidence between the endogenous and exogenous components of the circadian rhythm, and this lack could account for the observed decrease in rhythm amplitude during the night shift.

The phase delay of the purified data was only $1.7 \mathrm{~h}$ during the two night shifts. In agreement with this finding, earlier studies based on oral temperature data had already indicated that, in rapidly rotating threeshift work (1-2 nights in succession), the adjustment of the temperature rhythm was only marginal $(18,19)$. It is surprising therefore that, in a recent study of nurses working a greater number of successive night shifts (6), a similar methodology was used (nurses, continuous rectal temperature recording, masking effects removed), but the average phase delay during the same time period (two nights) was clearly greater, $4.6 \mathrm{~h}$.
Table 3. Stepwise regression analyses of the phase shift of the body temperature from the morning to the second night shift with the use of the cross-correlation methods with the raw and purified data. The studied individual factors explained $61 \%$ of the variation of the phase shift of the raw data (R-square 0.61 , total df $16, F=3.4, P<0.05$ ) and $46 \%$ of the variation of the phase shift of the purified data (R-square 0.46 , total df 16 $\mathrm{F}=3.7, \mathrm{P}<0.05)$. $(\mathrm{df}=$ degrees of freedom, $\mathrm{NS}=$ not significant, $\dot{\mathrm{V}}_{2}$ max $=$ maximal oxygen consumption)

\begin{tabular}{lll}
\hline & $F$ & P-value \\
\cline { 2 - 3 } & & \\
Phase shift of the raw data & & \\
Intercept & 9.58 & 0.05 \\
Neuroticism & 9.80 & 0.01 \\
Height & 7.01 & 0.05 \\
VO 2 max & 3.34 & $\mathrm{NS}$ \\
Diurnal type & 1.94 & $\mathrm{NS}$ \\
$\quad$ Introversion-extroversion & 0.7 & $\mathrm{NS}$ \\
Phase shift of the purified data & & \\
Intercept & 2.65 & $\mathrm{NS}$ \\
Diurnal type & 5.4 & 0.05 \\
Children & 3.38 & $\mathrm{NS}$ \\
Weight & 0.76 & $\mathrm{NS}$ \\
\hline
\end{tabular}

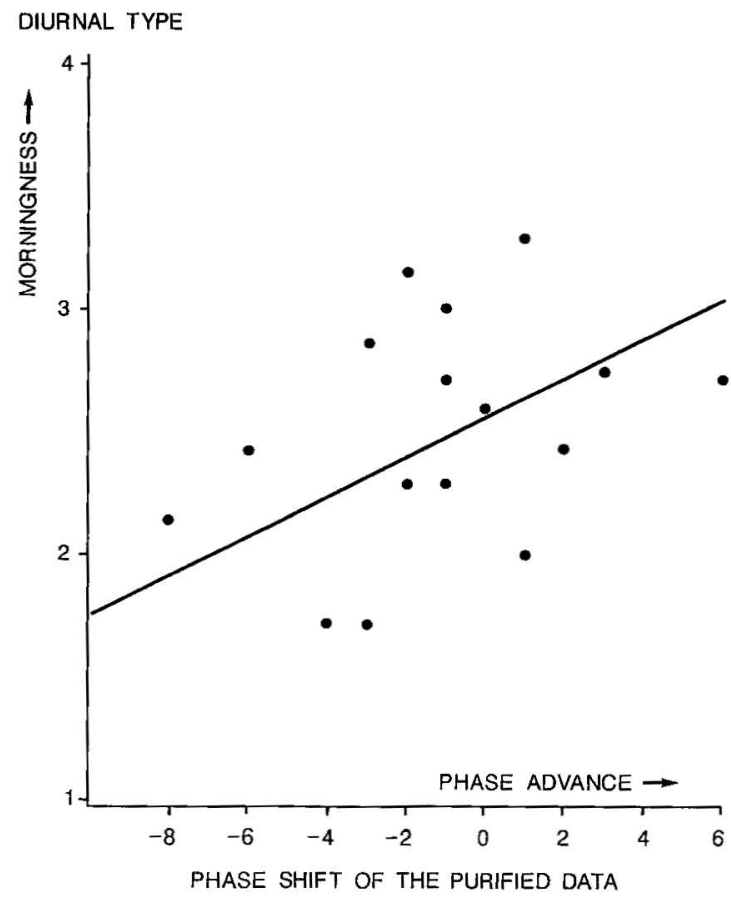

Figure 3. Correlation $(r=0.56, P<0.019)$ between diurnal type ( $4=$ extreme morningness, $1=$ extreme eveningness) and the phase shift of the purified data between the morning and second night shift.

Since the main difference between this study and that of Minors \& Waterhouse (6) appeared to be in the speed of rotation of the shift schedule, it seems possible that subjects on rapidly rotating shift schedules adjust their endogenous rhythmicity slower than do subjects with longer spans of night work. It is possible that a more rapid circadian adjustment may be beneficial only in slowly rotating shift systems, be- 
cause in rapidly rotating shift schedules circadian adjustment may be unnecessary or even detrimental - causing internal desynchronization and a decrease in shift-work tolerance. Therefore, some differences might exist between the subjects working these different schedules. In part these differences may be a form of natural selection, subjects who innately adjust more slowly being more suited to rapidly rotating shifts and, therefore, more likely to stay in them. But, in addition, there might be behavioral differences, those in slowly rotating shift systems adjusting their life-styles to a greater extent. That is, "motivation" to adjust might be more appropriate only with a slow rotation of shifts; by contrast, a better course of action for a subject on a rapidly rotating shift system would be to attempt to maintain a stable phasing of circadian rhythms (and one that is phased appropriately for a diurnal life-style) as much as possible $(20,21)$.

Diurnal type did not significantly explain the variations in the phase shifts of the raw data, but it was the only significant factor, explaining $32 \%$ of the variation of the phase shifts of the purified data. Morning "types" had a tendency towards slower circadian adjustment. It has been hypothesized that morningness is linked to a shorter endogenous period of circadian rhythmicity $(9,22)$. If true, this hypothesis would explain why morning types are not able to phase delay their circadian rhythms during night shifts as well as evening types. Although earlier field studies, which have not removed the masking effects of the circadian rhythm of body temperature, have found a similar relation between morningness and phase delay $(1,23,24)$, the interference due to masking effects might explain some earlier negative findings. (See, eg, references 9 and 25.) Our results suggest that the observed differences between morning and evening types in their ability to adjust to night work are not easily explained in terms of differences in behavior being initially responsible.

The degree of neuroticism was a significant predictor of the interindividual differences in the phase shift of the raw data, but it did not explain the phase shifts when the masking effects were removed. Vidacek et al (25) have shown that, although neuroticism correlates highly with the frequency of self-reported symptoms in shift work, in prospective studies, a person's neuroticism before starting shift work does not predict future shift-work tolerance. This finding demonstrated that poor shift-work tolerance might induce neuroticism. Since poor shift-work tolerance means, in most cases, sleep disturbances, the masking effects of sleep or other life-style differences could explain the observed relationship between neuroticism and faster adjustment of the raw, unpurified data.

Shift-work tolerance correlated highly with neuroticism, and it is probable that for this reason it was not selected into the regression model which explained the variation of the phase delays of the raw data. Shift-work tolerance correlated, however, with the phase delay of the raw data. Since shift-work tolerance did not correlate with the phase shift of the purified data, it can be speculated that the earlier observations of faster circadian adjustment of subjects with poor shift-work tolerance $(1,3,4)$ could again be due to the use of data from which masking effects on the circadian rhythm of body temperature had not been removed.

It seems clear that in future field studies of the circadian rhythm of body temperature, the masking effects on body temperature should be taken into account or, preferably, removed to increase the value of the results. In particular, the masking effects on body temperature should be removed before any definite conclusions about changes in the endogenous component of temperature circadian rhythmicity can be drawn.

\section{References}

1. Costa G, Lievore F, Casaletti G, Gaffuri E, Folkard $S$. Circadian characteristics influencing inter-individual differences in tolerance and adjustment to shiftwork. Ergonomics 1989;32:373-85.

2. Knauth P, Emde E, Rutenfranz J, Kiesswetter E, Smith P. Re-entrainment of body temperature in field studies of shiftwork. Int Arch Occup Environ Health 1981; 49:137-49.

3. Reinberg A, Vieux N, Ghata J, Chaumont AJ, Laporte A. Circadian rhythm amplitude and individual ability to adjust to shift work. Ergonomics 1978;21:763-6.

4. Reinberg A, Vieux N, Andlauer P, Guillet P, Nicolai A. Tolerance of shiftwork, amplitude of circadian rhythms and ageing. In: Reinberg A, Vieux N, Andlauer $P$, ed. Night and shiftwork: biological and social aspects. Oxford: Pergamon Press, 1981:341—54.

5. Minors DS, Waterhouse JM. Investigating the endogenous component of human circadian rhythms: a review of some simple alternatives to constant routines. Chronobiol Int 1992;9:55-78.

6. Minors DS, Waterhouse JM. Separating the endogenous and exogenous components of the circadian rhythm of body temperature during night work using some 'purification' models. Ergonomics 1993;36: 497-508.

7. Colquhoun WP, Condon R. Introversion-extroversion and the adjustment of the body-temperature rhythm to night work. In: Reinberg A, Vieux N, Andlauer P, ed. Night and shift work: biological and social aspects. Oxford: Pergamon Press, 1981:449-55.

8. Colquhoun WP, Folkard S. Personality differences in body-temperature rhythm, and their relation to its adjustment to night work. Ergonomics 1978;21:811-7.

9. Monk TH, Folkard S. Individual differences in shiftwork adjustment. In: Folkard S, Monk TH, ed. Hours of work: temporal factors in work-scheduling. Chichester: John Wiley, 1985:227-37.

10. Härmä M. Individual differences in tolerance to shiftwork: a review. Ergonomics 1993;36:101-10.

11. Otto H, Dillmann P, Knauth P, Rutenfranz J. Ein tragbarer digitaler Messwertspeicher mit hoher Auflösung fur Körpertemperaturen. Biomed Tech (Berlin) 1981; 26:9-12.

12. Nelson W, Tong YL, Lee JK, Halberg F. Methods for cosinor-rhythmometry. Chronobiologia 1979;6:30523.

13. Wever RA. Internal interactions within the human circadian system: the masking effect. Experientia 1985; 
$41: 332-342$.

14. Torsvall L, Åkerstedt T. A diurnal type scale: construction, consistency and validation in shift work. Scand J Work Environ Health 1980;6:283-90.

15. Eysenck HJ. A short questionnaire for the measurement of two dimensions of personality. J Appl Psychol 1958;42:14-7.

16. Knauth $\mathrm{P}, \mathrm{Härmä} \mathrm{M}$. The relation of shift work tolerance to the circadian adjustment. Chronobiol Int 1992; $9: 46-54$.

17. Andersen KL, Shephard R, Denolin H, Varnauskas E, Masironi R. Fundamentals of exercise testing. Geneva: Work Health Organization, 1971.

18. Smith P. A study of weekly and rapidly rotating shift workers. Int Arch of Occup and Environ Health 1979; 46:111-25.

19. Dahlgren K. Adjustment of circadian rhythms to rapidly rotating shift work - a field study of two shift systems. In: Reinberg A, Vieux N, Andlauer P, ed. Night and shift work: biological and social aspects. Oxford: Pergamon Press, 1981:357-64.

20. Folkard S. Is there a "best compromise" shift system?
Ergonomics 1992;35:1453-63.

21. Wilkinson RT. How fast should the night shift rotate? Ergonomics 1992;35:1425-46.

22. Kerkhof GA. Inter-individual differences in the human circadian system: a review. Biol Psychol 1985;20:83112.

23. Breithaupt H, Hildebrandt G, Dohre D, Josch R, Sieber $U$, Werner $M$. Tolerance to shift of sleep as related to the individual's circadian phase position. Ergonomics 1978;21:767-74.

24. Östberg O. Circadian rhythms of food intake and oral temperature in "Morning" and "Evening" groups of individuals. Ergonomics 1973;16:203-9.

25. Vidacek S, Kaliterna L, Radosevic-Vidacek B. Predictive validity of individual difference measures for health problems in shiftworkers: preliminary results. In: Oginski A, Pokorski J, Rutenfranz J, ed. Contemporary advances in shiftwork research. Krakow: Medical Academy, 1987:99-106.

Received for publication: 26 May 1993 